\title{
Quantitative trait loci mapping for kernel row number using chromosome segment substitution lines in maize
}

\author{
F. Li, H.T. Jia, L. Liu, C.X. Zhang, Z.J. Liu and Z.X. Zhang \\ National Key Laboratory of Crop Genetic Improvement, \\ Huazhong Agricultural University, Wuhan, China \\ Corresponding author: Z.X. Zhang \\ E-mail: zuxinzhang@mail.hzau.edu.cn
}

Genet. Mol. Res. 13 (1): 1707-1716 (2014)

Received April 9, 2013

Accepted September 3, 2013

Published January 17, 2014

DOI http://dx.doi.org/10.4238/2014.January.17.1

\begin{abstract}
Unveiling the genetic architecture of grain yield and yieldrelated traits is useful for guiding the genetic improvement of crop plants. Kernel row number (KRN) per ear is an important yield component, which directly affects the grain yield of maize. In this study, we constructed a set of 130 chromosome segment substitution lines (CSSLs), using Nongxi531 as the donor parent and H21 as recipient parent, by continuous backcrossing and selfing. In total, 11 quantitative trait loci (QTL) were detected for KRN by stepwise regression under 3 environmental settings, with 9.87-19.44\% phenotypic variation being explained by a single QTL. All 11 QTL were also detected by single-factor ANOVA across the 3 environments tested. Of these 11 QTL, 4 were identified across more than 2 environments, indicating that they are authentically expressed under different environments to control the formation and development of KRN in female maize inflorescences. The CSSLs harbored a greater number of favorable alleles for KRN compared to the H21 line, and could be employed as improved H21 lines in maize breeding programs.
\end{abstract}

Key words: Chromosome segment substitution lines;

Maize (Zea mays L.); Kernel row number; Quantitative trait loci 


\section{INTRODUCTION}

Maize is the most important source of calories for humans, in addition to providing an important source of energy, because its abundant carbohydrates may be converted into ethanol. As a result, there is intense pressure to improve maize grain yields to meet the rapidly expanding global demands for maize-derived food, feed, and fuel (Rosegrant et al., 2001). To improve the grain yield of maize, better knowledge about the genetic basis of yield traits is the premise for establishing breeding program. However, maize grain yield is a complex trait, which is genetically controlled by numerous quantitative trait loci (QTL) with minor genetic effect, which is also compounded by extensive interactions among QTL and between QTL and the environments $(\mathrm{Xu}, 2010)$. Thus, this genetic complexity has inhibited our ability to develop a good understanding about the genetic basis of maize, leading to minimal progress towards improving maize grain yields through the direct manipulation of QTL. An alternative strategy for elucidating the genetic architecture of grain yield is dissecting complex grain yield traits into relatively simple yield components, and then combining all of the genetic information underlying these yield components to elucidate the genetic basis of grain yield. Kernel row number (KRN) is one of the important yield components that show a significantly positive correlation with grain yield. Therefore, the identification and cloning of QTL associated with KRN is particularly useful for determining the genetic basis and guiding the genetic improvement of grain yield of maize.

Classical QTL mapping populations are derived from bi-parental crossing, such as $\mathrm{F}_{2: 3}$ families, backcross populations, recombinant inbred lines, and double haploids. Chromosome segment substitution line (CSSL) or introgression line (IL) populations may be used as a superior way for the identification and fine-scale mapping of QTL. The first set of CSSLs was constructed and employed for the fine-scale mapping of fruit size QTL fw2.2 in tomato (Paterson et al., 1990; Eshed and Zamir, 1995; Frary et al., 2000). Subsequently, many CSSL populations have been reported in a diverse range of plant species, such as rice (Li et al., 2005; Zeng et al., 2006; Marzougui et al., 2012), soybean (Yamanaka et al., 2005), and barley (Von Korff et al., 2004). Szalma et al. (2007) constructed a set of ILs in maize to detect QTL underlying maize flowering time, plant height, and ear height, by introgressing chromosome segments of TX303 to the B73 genome. In another study, Bai et al. (2010) detected a set of QTL for plant height and ear height using 98 ILs derived by Zong 3 x HB522, from which it was confirmed that the non-additive action was the major genetic basis of both plant height and ear height. Most of the initial studies showed that CSSL or IL populations are more effective for QTL identification compared to populations derived from bi-parental $\mathrm{F}_{2}$. This difference arises because of the absence of a complex genetic background and because the introgressed segments are the major source of genetic variation compared to the recipient parent (Paterson et al., 1990; Kaeppler, 1997; Szalma et al., 2007).

More than 100 QTL have been mapped on the whole genome of maize for KRN over the last 20 years, using different mapping populations subject to different environments (Veldboom et al., 1994; Ribaut et al., 1997; Upadyayula et al., 2006; Ma et al., 2007; Lu et al., 2010; Brown et al., 2011; Liu et al., 2012). Lu et al. (2010) detected 13 QTL for KRN in 7 environments using 397 $\mathrm{F}_{2: 3}$ families with a major QTL being detected on chromosome 7 across all environments. Zhao et al. (2011) identified 10 QTL for KRN in 2 sets of introgression lines, with 2 of the QTL being detected across 2 environments. More recently, Brown et al. (2011) surveyed the inflorescence traits of maize by linkage analysis and genome-wide association analysis in a NAM population, which led to the identification of $36 \mathrm{KRN}$-associated QTL and 261 SNPs. In this study, we constructed a 
CSSL population by continuously backcrossing and self-crossing, in combination with molecular marker assisted selection. As a result, a set of QTL for KRN was identified under 3 environments.

\section{MATERIAL AND METHODS}

\section{Population construction}

Two elite inbred lines Nongxi531 and H21 were employed as donor and recurrent parent, respectively. Nongxi531 and H21 had significantly different genetics and phenotypes. Nongxi531 is the female parent of an elite hybrid Nongdan5 in China. It has 18-20 kernel rows and a high seed-producing percentage. Genetically, this inbred line belongs to the Reid heterotic group. In comparison, H21 exhibits generally high combining ability and drought resistance, but produces an ear with just 8-12 kernel rows. The F1 individuals of $\mathrm{H} 21 \mathrm{x}$ Nongxi531 were continuously backcrossed with $\mathrm{H} 21$ to $\mathrm{BC}_{4} \mathrm{~F}_{1}$. A total of $526 \mathrm{BC}_{4} \mathrm{~F}_{1}$ individuals were genotyped using 176 SSR markers. As a result, 130 individuals with fewer donor segments and higher genome coverage were selected to self-cross into $\mathrm{BC}_{4} \mathrm{~F}_{4}$, to construct a set of 130 CSSLs on an $\mathrm{H} 21$ genetic background (referred to as H21CSSLs).

\section{Phenotype and genotype identification}

The H21CSSLs was planted in 3 different locations (i.e., 3 different environments) in China between 2010 and 2011 [ $\left(\right.$ Sanya $\left(18^{\circ} \mathrm{N}, 109^{\circ} \mathrm{E}\right)$ in 2010 , and Wuhan $\left(30^{\circ} \mathrm{N}, 114^{\circ} \mathrm{E}\right)$ and Baoding $\left(38^{\circ} \mathrm{N}, 115^{\circ} \mathrm{E}\right)$ in 2011$\left.)\right]$ using a random block design with 3 replications. Twelve plants of each line were planted in a plot with $3.0 \mathrm{~m}$ long rows, and the spacing between rows was $0.6 \mathrm{~m}$. H21 was used as a control line, and was planted on the blocks in the first, center and end row, respectively. Seven to 9 plants were selected from each line to evaluate KRN. The plants were scored based on the number of rows of kernels in the middle of ear.

Total genomic DNA from maize leaves was extracted using a CTAB procedure (Saghai-Maroof et al., 1984). DNA concentration was estimated using a NanoDrop ${ }^{\circledR}$ spectrophotometer, and then adjusted to $10 \mathrm{ng} / \mu \mathrm{L}$. The PCR mixture contained template $3 \mu \mathrm{L} \mathrm{DNA}$ (about $30 \mathrm{ng}), 1 \mu \mathrm{L} 10 \mathrm{X}$ buffer, $0.75 \mu \mathrm{L} \mathrm{Mg}^{2+}, 0.25 \mu \mathrm{L}$ dNTP $(2.5 \mathrm{mM}$ each $), 0.06 \mu \mathrm{L}$ Taq $(5 \mathrm{U} / \mu \mathrm{L})$,

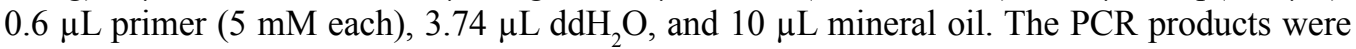
mixed with $10 \mu \mathrm{L}$ loading buffer, and were then electrophoresed on $6 \%$ denaturing polyacrylamide gel using $1 \mathrm{X}$ TBE running buffer. The DNA bands were visualized by silver staining.

Using the IBM 2008 neighbor genetic map (www.maizegdb.org), 691 markers that are uniformly distributed on the maize genome were selected to evaluate genetic diversity between Nongxi531 and H21. A total of 307 ( 44\%) polymorphic markers were identified. Of these, 176 markers that are able to amplify stable and well distinguishable bands were used to genotype $\mathrm{BC}_{4} \mathrm{~F}_{1}$ individuals. The graphical genotypes were used to evaluate the number, size, and location of introgressed segments, the percentage of the recurrent genome coverage (PRC), and the percentage of the recurrent genome (PRG) of each CSSL line (Young and Tanksley, 1989).

\section{Data analysis}

PRG was estimated based on the formula developed by Hospital and Charcosset (1997) 
and Young and Tanksley (1989), which is calculated as $\mathrm{G}(\mathrm{g})=[\mathrm{L}+\mathrm{X}(\mathrm{g})] /(2 \mathrm{~L})=1 / 2+(1 / 2)$ $(\mathrm{X}(\mathrm{g}) / \mathrm{L})$, where $G(\mathrm{~g})$ is the percentage of recurrent genome coverage in the g-generation, $X(\mathrm{~g})$ is the number of molecular markers that are located on the genotype of the recurrent parent in the g-generation, and $L$ is to the total number of molecular markers. Two-factor ANOVA (analysis of variance) was conducted using the software SAS 9.1 (SAS Institute, 2003), and was used to estimate phenotype variance, correlation coefficient, and heritability. Marker-KRN associations were calculated by the method of single-factor ANOVA, and the mean values of each genotype and $\mathrm{P}$ values were obtained for each marker locus. In addition, the QTL for KRN were identified by a likelihood ratio test based on stepwise regression by QTL ICIMapping V3.0 (Wang et al., 2006; Li et al., 2007), with 1000 times the permutation tests for the threshold logarithm of the odds (LOD). In general, the genetic effect of lines harboring a single QTL substitutive segment is equivalent to its additive effect; however, the genetic effect of double QTL pyramiding lines is composed of the additive effects of every CSSL harboring the substitutive segment from a single QTL and the interaction between these 2 QTL (Zhao et al., 2012).

\section{RESULTS}

\section{H21CSSLs PRG}

The PRG of the H21CSSLs ranged from $78.7 \%$ to $99.4 \%$, with most having a PRG of $94.0 \%$ to $97.0 \%$ (96/130 lines) based on the genotype, by using 176 markers spread through the whole genome. The PRG of 2 CSSLs was more than $99.0 \%$, while the PRG of 6 CSSLs was less than $92.0 \%$ (Figure 1). A total of 1277 donor segments were introgressed in the H21CSSLs, with about 10 segments per line. The accumulative size of the introgressed donor segments was $\sim 90486.0 \mathrm{cM}$, using the IBM 2008 neighbor genetic map as a reference. The size of unique introgressed segments was $\sim 6130.0 \mathrm{cM}$, and covered $85.8 \%$ of the $\mathrm{H} 21$ genome in total. Chromosomes 3 and 10 were completely covered by the segments, while chromosome 8 was only $66.7 \%$ covered.

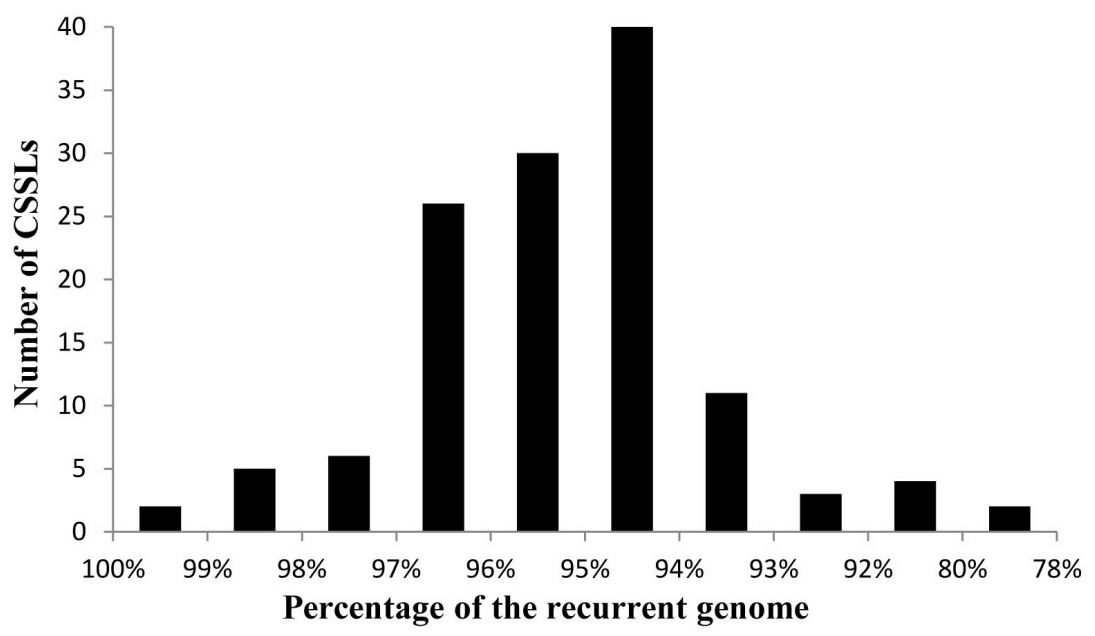

Figure 1. Percentage of the recurrent genome of H21CSSLs. 


\section{Phenotypic analysis of H21CSSLs}

A total of 130 lines of H21CSSLs and both parents (Nongxi531 and H21) were evaluated for KRN in 3 environments. The phenotype was significantly correlated in 3 environments (Table 1), while the broad-sense heritability for KRN was $75.40 \%$. The means of the KRNs in the H21CSSLs ranged from 9.82 to 11.45 rows in 3 environments, with no significant difference being found compared to $\mathrm{H} 21$. This result indicated the presence of a strong background effect, because of the high PRG of H21CSSLs. However, KRN covered from 8 to 18 rows in the H21CSSLs, with differences in the KRN being significant among inbred lines (Table 2). This result indicated that the introgressed donor segments of different lines had a markedly different phenotypic effect on KRN. The phenotype data of the KRN in the H21CSSLs showed continuous variation, and fitted the normal distribution by a Skewness-Kurtosis test, revealing a quantitative trait characteristic, which confirmed that this population was appropriate for QTL mapping.

\begin{tabular}{lcc}
\multicolumn{2}{l}{ Table 1. Correlation analysis of kernel row number among environments. } & \\
\hline Environment & Sanya & Wuhan \\
\hline Wuhan & $0.51^{* *}$ & $0.65^{* *}$ \\
\hline Baoding & $0.56^{* *}$ & \\
\hline ** Significance at $\mathrm{P}<0.001$. & &
\end{tabular}

Table 2. Kernel row number phenotype of $\mathrm{H} 21$ and CSSL population under different environments.

\begin{tabular}{|c|c|c|c|c|c|c|}
\hline \multirow[t]{2}{*}{ Environment } & \multirow{2}{*}{$\begin{array}{c}\mathrm{H} 21 \\
\text { Means }\end{array}$} & \multicolumn{5}{|c|}{ CSSLs } \\
\hline & & Means & Range & $\mathrm{CV} \%$ & Skewness & Kurtosis \\
\hline Sanya & $9.5 \pm 1.35$ & $9.8 \pm 1.47$ & $8-16$ & 0.15 & 0.50 & 0.19 \\
\hline Wuhan & $11.2 \pm 1.04$ & $11.5 \pm 1.39$ & $8-16$ & 0.12 & 0.08 & 0.13 \\
\hline Baoding & $10.5 \pm 1.60$ & $10.9 \pm 1.58$ & $8-18$ & 0.15 & 0.22 & 0.05 \\
\hline
\end{tabular}

\section{QTL mapping}

We assumed that the QTL that were mapped to the same genomic regions in different environments represented the same QTL. A total of 47 QTL for KRN were detected by single-factor ANOVA below the threshold of P $\leq 0.001$. Of these 47 QTL, 6 were detected in 3 environments and 9 were detected in 2 environments. However, only 11 QTL for KRN were detected by single-factor ANOVA, and were repeatedly detected by stepwise regression analysis at $\mathrm{P} \leq 0.01$. Eleven QTL were located on chromosomes 2, 3, 4, 6, 7, 8, and 9. The phenotypic variation explained (PVE) by a single QTL ranged from $9.65 \%$ to $19.44 \%$ (Table 3 ). Both $q K R N 7 a$ and $q K R N 7 b$ were detected with large additive effects across 3 environments, which could be used to increase number of kernel rows of the maize plant to increase by 1.4 and 1.5 rows, respectively, compared to $\mathrm{H} 21$. Both $q K R N 9 a$ and $q K R N 9 b$ were repeatedly detected from the Wuhan and Baoding maize plots, with $19.94 \%$ and $9.91 \%$ PVE, respectively. In addition, all of the QTL showed positive additive effects, except for $q K R N 8$, indicating that the majority of introgressed segments from Nongxi531 harbor favorable alleles compared to the H21 counterparts. These QTL could be used to improve the KRN of H21. 


\begin{tabular}{|c|c|c|c|c|c|c|c|c|c|}
\hline Environment & QTL & Marker & Bin & LOD & PVE (\%) & Add & $<1 \mathrm{M}$ & $1-5 \mathrm{M}$ & $5-10 \mathrm{M}$ \\
\hline \multirow{8}{*}{ Sanya } & $q K R N 2$ & bnlg2248 & 2.03 & 3.94 & 12.22 & 1.27 & & & $z f l 2$ \\
\hline & $q K R N 4 a$ & umc 2410 & 4.02 & 3.50 & 11.76 & 1.78 & & & \\
\hline & $q K R N 4 b$ & umc1086 & 4.08 & 4.01 & 12.51 & 1.80 & & & \\
\hline & $q K R N 4 c$ & umc2011 & 4.01 & 4.66 & 14.26 & 0.99 & & & \\
\hline & $q K R N 6$ & umc1520 & 6.06 & 3.63 & 12.27 & 1.79 & & & Tsh1 \\
\hline & $q K R N 7 a$ & umc1270 & 7.01 & 5.71 & 15.24 & 1.40 & & & \\
\hline & $q K R N 7 b$ & umc1125 & 7.04 & 4.67 & 16.25 & 1.45 & $r a 3$ & $b d l$ & dlf1 \\
\hline & $q K R N 8$ & umc1005 & 8.08 & 2.57 & 9.82 & -0.38 & & & \\
\hline \multirow[t]{4}{*}{ Wuhan } & $q K R N 7 a$ & umc1270 & 7.01 & 2.66 & 9.92 & 0.91 & & & \\
\hline & $q K R N 7 b$ & umc1125 & 7.04 & 3.02 & 11.71 & 0.99 & $r a 3$ & $b d l$ & dlf1 \\
\hline & $q K R N 9 a$ & umc2519 & 9.06 & 5.84 & 19.44 & 0.51 & & & \\
\hline & $q K R N 9 b$ & bnlg 1525 & 9.07 & 3.34 & 12.81 & 0.42 & & & \\
\hline \multirow[t]{5}{*}{ Baoding } & $q K R N 3$ & umc2369 & 3.03 & 3.43 & 11.97 & 1.94 & & $\mathrm{Cg} 1, \mathrm{ra} 2$ & \\
\hline & $q K R N 7 a$ & umc1270 & 7.01 & 4.49 & 15.97 & 1.54 & & & \\
\hline & $q K R N 7 b$ & umc1125 & 7.04 & 2.60 & 9.65 & 1.20 & $r a 3$ & $b d l$ & $d l f 1$ \\
\hline & $q K R N 9 a$ & umc2519 & 9.06 & 2.84 & 9.91 & 0.49 & & & \\
\hline & $q K R N 9 b$ & bnlg 1525 & 9.07 & 2.56 & 9.87 & 0.50 & & & \\
\hline
\end{tabular}

$\overline{\mathrm{PVE}}=$ phenotype variation explained by QTL. Add = additive effect. $<1 \mathrm{M}$ shows the physical distance between QTL and cloned mutant gene is less than 1 million base pairs. ra3 = ramosa 3 (Satoh-Nagasawa et al., 2006). $b d 1=$ branched silkless 1. (Chuck et al., 2002). dlf1 = delayed flowering 1. (Muszynski et al., 2006). tsh1= tassel sheath 1. (Whipple et al., 2010). zfl2 = zea floricaula/leafy 2. (Bomblies et al., 2003). Cg1 = Corngrass 1. (Chuck et al., 2007). ra2 = ramosa 2 (Bortiri et al., 2006).

\section{Phenotypic effects of CSSLs harboring donor segments at QTL}

The lines that had donor segments at QTL for KRN were selected from the H21CSSLs to evaluate the phenotypic effect of the donor segment across 3 environments. Most of the selected lines showed significantly higher KRN compared to H21, indicating that the phenotypic effects of donor alleles might differ to the H21 counterparts (Table 4). The lines that had donor segments at $q K R N 2, q K R N 4 c, q K R N 7 a, q K R N 7 b, q K R N 9 a$, and $q K R N 9 b$ could be used to add about 2 kernel rows to the maize plant compared to $\mathrm{H} 21$. The lines with donor segments at $q K R N 4 a, q K R N 4 b$, and $q K R N 6$ could add almost 3 rows to the maize kernel. In contrast, the donor segment at $q K R N 8$ decreased KRN compared to H21. Thus, the majority of donor segments at these QTL harbor favorable alleles for enhancing KRN. This finding might be explained by Nongxi531 being a high-KRN inbred line. However, some lines contained more than 2 donor segments, resulting in a strongly positive effect of the donor segment harboring QTL for KRN, which might have been caused by the by complex interaction among donor segments or between the donor segments and the $\mathrm{H} 21$ genome.

Table 4. Kernel row number (KRN) of those lines with donor segments at quantitarive trait loci (QTL).

\begin{tabular}{|c|c|c|c|}
\hline \multirow[t]{2}{*}{ QTL } & \multirow[t]{2}{*}{ Number of SSLs } & \multicolumn{2}{|c|}{ KRN } \\
\hline & & CSSL & $\mathrm{H} 21$ \\
\hline$q K R N 2$ & 2 & $12.3 * *$ & 10.1 \\
\hline$q K R N 3$ & 1 & $14.6 * *$ & 10.1 \\
\hline$q K R N 4 a$ & 1 & $13.3 * *$ & 10.1 \\
\hline$q K R N 4 b$ & 1 & $13.3 * *$ & 10.1 \\
\hline$q K R N 4 c$ & 4 & $11.8 * *$ & 10.1 \\
\hline$q K R N 6$ & 1 & $13.3 * *$ & 10.1 \\
\hline$q K R N 7 a$ & 2 & $12.6 * *$ & 10.1 \\
\hline$q K R N 7 b$ & 2 & $12.7 * *$ & 10.1 \\
\hline$q K R N 8$ & 19 & 9.2 & 10.1 \\
\hline$q K R N 9 a$ & 10 & $12.4 * *$ & 10.1 \\
\hline$q K R N 9 b$ & 8 & $12.4 * *$ & 10.1 \\
\hline
\end{tabular}

**Significance at $\mathrm{P}<0.001$. 


\section{Interaction effect between QTL for KRN}

Under a purely additive-dominance model, genotypic value with multiple loci may be predicted by the genetic effect of single factor. However, gene-gene interaction is an important genetic component of complex traits. We selected the lines that harbored 2 QTL introgression segments (double QTL pyramiding lines) from the H21CSSLs, to detect the interactions of 2-locus QTL by comparing double QTL pyramiding lines with single QTL harboring lines and the recurrent parent H21. Four pairs of significant 2-locus QTL interactions were identified (Table 5). For instance, $q K R N 4 c$ showed interactions with 3 QTL; namely, $q K R N 7 a, q K R N 9 a$, and $q K R N 9 b$. In addition, $q K R N 9 a$ also significantly interacted with $q K R N 9 b$. The interaction effect of $q K R N 4 c$ with $q K R N 9 a$ was 0.86 rows, when the signal-locus additive effect was 0.61 rows and 0.98 rows, respectively; thus, the KRN of the double QTL pyramiding line increased the maize kernel by 2.45 rows compared to $\mathrm{H} 21$. Similarly, the interaction effect of $q K R N 4 c$ with $q K R N 7 a$ was negative $(-0.27$ row $)$, while the signal-loci additive effect was positive ( 0.14 rows and 1.21 rows, respectively); thus, the KRN of the double QTL pyramiding line increased the number of kernel rows by 1.07 compared to H21. This result implied that the genetic effect of the double QTL pyramiding line was not the result of a simple accumulation of the 2 single-locus additive effect.

Table 5. Interaction effect among major QTL for KRN.
\begin{tabular}{lccccc}
\hline QTL1 & QTL2 & $\mathrm{A} 1$ & $\mathrm{~A} 2$ & $\mathrm{~A} 1 \mathrm{~A} 2$ & LOD \\
\hline$q K R N 4 c$ & $q K R N 7 a$ & 0.14 & 1.21 & -0.27 & 6.26 \\
$q K R N 4 c$ & $q K R N 9 a$ & 0.61 & 0.98 & 0.86 & 6.86 \\
$q K R N 4 c$ & $q K R N 9 b$ & 0.59 & 0.82 & 0.84 & 7.93 \\
$q K R N 9 a$ & $q K R N 9 b$ & 0.38 & 0.19 & -0.17 & 6.81 \\
\hline
\end{tabular}

A1 indicates the additive effect of QTL1, A2 indicates the additive effect of QTL2, A1 A2 indicates the additive by additive effect.

\section{DISCUSSION}

\section{Effective detection of QTL for KRN using the H21CSSL population}

A total of 11 QTL for KRN were detected by both single-factor ANOVA and stepwise regression using QTL ICIMapping V3.0. Additional minor QTL were detected by single-factor ANOVA in the H21CSSL population, indicating that the CSSL population could be used to identify both major and minor QTL, because of the high similarity in the genetic background among CSSLs and between each CSSL and H21; thus, confirming the high power of QTL detection by this line. QTL for KRN that were detected in this study were highly consistent with the results of previous studies. For example, a QTL for KRN that was detected by Yan et al. (2006) in the bnlg1064-umc118 interval was similar to qKRN2. In addition, another QTL in umc1173-umc2286 was similar to $q K R N 4 b$. A QTL for KRN detected by Tang et al. (2007) in umc2101-bnlg1523 was similar to $q K R N 3$. In particular, both $q K R N 7 b$ and $q K R N 9 b$ identified in the current study were also found by Lu et al. (2010) across 7 environments. Zhao et al. (2012) mapped a total of 10 QTL for KRN from 2 introgression line populations, and found that the QTL close to umc 1185 was the same as $q K R N 2$. These QTL, which were detected in different populations, indicated that these loci are probably important for controlling the differentiation and formation of 
KRN in the female inflorescence of maize. In addition, the identification of these QTL provides the basis from which to conduct fine-scale mapping and gene cloning studies.

\section{Differences in loci controlling natural variation of KRN compared to those uncovered using mutagenesis}

Twenty-seven developmental genes for inflorescence have been cloned in maize. Most of these developmental genes are involved in loss-of-function alleles, caused by transposon or chemical mutagenesis (Brown et al., 2011). Upadyayula et al. (2006) identified a set of QTL for tassel branch number, central spike spikelet pair density, kernel number density, kernel row number, kernel number per row, and cob diameter. Some of these parameters were co-located with genes that are known to affect the development of maize inflorescences, such as ramosal (Vollbrecht et al., 2005), ramosa2, thick tassel dwarf1 (Bommert et al., 2005), and fasciated ear2 (Taguchi-Shiobara et al., 2001); however, other genes in the QTL regions were unknown. We found that only a few cloned mutant genes were closely linked with the QTL detected for KRN in this investigation. For example, ramosa3, which is a mutant gene regulating inflorescence branching, is co-localized with $q K R N 7 b$. In addition, 7 mutant genes were located within a $10 \mathrm{Mb}$ region of the QTL (Table 3). Thus, we suggest that loci controlling natural variation of KRN might be identified by mutants in maize. QTL mapping could be used to identify many new genes that contribute to the formation and development of female inflorescence in maize. This suggestion is supported by the results of Brown et al. (2011) from GWAS using a NAM population.

\section{Improvement of H21 KRN by the chromosome segment of Nongxi531}

$\mathrm{KRN}$ is an important target trait in breeding, and plays a key role in the formation of maize seeds for cereal crop production. H21 is related to Huangzao4, forming an elite inbred line with just 10-12 kernel rows. In this study, the genome segment of Nongxi531, which exhibits 18-20 kernel rows and a high seed-producing percentage, was introgressed into the H21 genome to improve the KRN of H21. Because the Nongxi531 genome contains most of the favorable alleles for KRN, the KRN of many CSSLs tended to increase in comparison to H21. However, the morphological traits of CSSLs were highly similar to H21, because of the high PRG of most lines in the H21CSSLs. This result indicates that the improvement of the H21 KRN was achieved by strict background selection using marker-assisted selection.

Moreover, a phenotype comparison between H21 (q1q1q2q2) and CSSLs with 3 genotypes (Q1Q1q2q2, q1q1Q2Q2, and Q1Q1Q2Q2) revealed that the KRN of the double QTL pyramiding lines significantly increased compared to H21. In addition, the genetic effects of some double QTL pyramiding were larger compared to the sum of the additive effect of the 2 single-loci. Therefore, QTL pyramiding remains an effective strategy for improving KRN. Of note, some QTL were found to significantly affect KRN by additive interaction (Table 5). Furthermore, many loci that were detected by single factor ANOVA were not detected by stepwise regression analysis in our study because their minor genetic effects may also affect the KRN phenotype through the interaction with other loci or environments. Thus, we suggest that epistasis should be used as an important genetic component for estimating the breeding value of QTL pyramidal lines. 


\section{ACKNOWLEDGMENTS}

Research supported by the National Basic Research Program of China (\#2009CB118400 and \#2014CB138203) and the National Natural Science Foundation of China (\#31271738).

\section{REFERENCES}

Bai W, Zhang H, Zhang ZX, Teng F, et al. (2010). The evidence for non-additive effect as the main genetic component of plant height and kernel height in maize using introgression line populations. Plant Breeding 129: 376-384.

Bomblies K, Wang RL, Ambrose BA, Schmidt RJ, et al. (2003). Duplicate FLORICAULA/LEAFY homologs zfl and zfl2 control inflorescence architecture and flower patterning in maize. Development 130: 2385-2395.

Bommert P, Lunde C, Nardmann J, Vollbrecht E, et al. (2005). Thick tassel dwarfl encodes a putative maize ortholog of the Arabidopsis CLAVATA1 leucine-rich repeat receptor-like kinase. Development 132: 1235-1245.

Bortiri E, Chuck G, Vollbrecht E, Rocheford T, et al. (2006). ramosa2 encodes a LATERAL ORGAN BOUNDARY domain protein that determines the fate of stem cells in branch meristems of maize. Plant Cell 18: 574-585.

Brown PJ, Upadyayula N, Mahone GS, Tian F, et al. (2011). Distinct genetic architectures for male and female inflorescence traits of maize. PLoS Genet. 7: e1002383.

Chuck G, Muszynski M, Kellogg E, Hake S, et al. (2002). The control of spikelet meristem identity by the branched silkless 1 gene in maize. Science 298: 1238-1241.

Chuck G, Cigan AM, Saeteurn K and Hake S (2007). The heterochronic maize mutant Corngrass1 results from overexpression of a tandem microRNA. Nat. Genet. 39: 544-549.

Eshed Y and Zamir D (1995). An introgression line population of Lycopersicon pennellii in the cultivated tomato enables the identification and fine mapping of yield-associated QTL. Genetics 141: 1147-1162.

Frary A, Nesbitt TC, Grandillo S, Knaap E, et al. (2000). fw2.2: a quantitative trait locus key to the evolution of tomato fruit size. Science 289: 85-88.

Hospital F and Charcosset A (1997). Marker-assisted introgression of quantitative trait loci. Genetics 147: 1469-1485.

Kaeppler SM (1997). Quantitative trait locus mapping using sets of near-isogenic lines: relative power comparisons and technical considerations. Theor. Appl. Genet. 95: 384-392.

Li HH, Ye GY and Wang JK (2007). A modified algorithm for the improvement of composite interval mapping. Genetics 175: 361-374.

Li ZK, Fu BY, Gao YM, Xu JL, et al. (2005). Genome-wide introgression lines and their use in genetic and molecular dissection of complex phenotypes in rice (Oryza sativa L.). Plant Mol. Biol. 59: 33-52.

Liu RX, Jia HT, Cao XL, Huang J, et al. (2012). Fine mapping and candidate gene prediction of a pleiotropic quantitative trait locus for yield-related trait in Zea mays. PLoS ONE 7: e49836.

Lu M, Xie CX, Li XH and Hao ZF (2010). Mapping of quantitative trait loci for kernel row number in maize across seven environments. Molecular Breeding 28: 143-152.

Ma XQ, Tang JH, Teng WT, Yan JB, et al. (2007). Epistatic interaction is an important genetic basis of grain yield and its components in maize. Molecular Breeding 20: 41-51.

Marzougui S, Sugimoto K, Yamanouchi U, Shimono M, et al. (2012). Mapping and characterization of seed dormancy QTLs using chromosome segment substitution lines in rice. Theor. Appl. Genet. 124: 893-902.

Muszynski MG, Dam T, Li B, Shirbroun DM, et al. (2006). Delayed flowering1 Encodes a basic leucine zipper protein that mediates floral inductive signals at the shoot apex in maize. Plant Physiol. 142: 1523-1536.

Paterson AH, DeVerna JW, Lanini B and Tanksley SD (1990). Fine mapping of quantitative trait loci using selected overlapping recombinant chromosomes, in an interspecies cross of tomato. Genetics 124: 735-742.

Ribaut JM, Jiang C, Gonzalez-de-Leon D and Edmeades GO (1997). Identification of quantitative trait loci under drought conditions in tropical maize. 2. Yield components and marker-assisted selection strategies. Theor. Appl. Genet. 94: 887-896.

Rosegrant MW, Paisner M, Meijer S and Witcover J (2001). Global Food Projections to 2020: Emerging Trends and Alternative Futures. International Food Policy Research Institute, Washington D.C.

Saghai-Maroof MA, Soliman KM, Jorgensen RA and Allard RW (1984). Ribosomal DNA spacer-length polymorphisms in barley: mendelian inheritance, chromosomal location, and population dynamics. Proc. Natl. Acad. Sci. U. S. A. 81: 8014-8018.

SAS Institute (2003). SAS Proprietary Software Release 9.1. SAS Institute Inc, Cary.

Satoh-Nagasawa N, Nagasawa N, Malcomber S, Sakai H, et al. (2006). A trehalose metabolic enzyme controls inflorescence 
architecture in maize. Nature 441: 227-230.

Szalma SJ, Hostert BM, Ledeaux JR, Stuber CW, et al. (2007). QTL mapping with near-isogenic lines in maize. Theor. Appl. Genet. 114: 1211-1228.

Taguchi-Shiobara F, Yuan Z, Hake S and Jackson D (2001). The fasciated ear2 gene encodes a leucine-rich repeat receptor-like protein that regulates shoot meristem proliferation in maize. Genes Dev. 15: 2755-2766.

Tang JH, Yan JB, Ma XQ, Teng WT, et al. (2007). Genetic dissection for grain yield and its components using an "Immortalized F2 Population" in maize. Acta Agron. Sin. 33: 1299-1303.

Upadyayula N, da Silva HS, Bohn MO and Rocheford TR (2006). Genetic and QTL analysis of maize tassel and ear inflorescence architecture. Theor. Appl. Genet. 112: 592-606.

Veldboom LR, Lee M and Woodman WL (1994). Molecular marker-facilitated studies in an elite maize population: I. Linkage analysis and determination of QTL for morphological traits. Theor. Appl. Genet. 88: 7-16.

Vollbrecht E, Springer PS, Goh L, Buckler ES, et al. (2005). Architecture of floral branch systems in maize and related grasses. Nature 436: 1119-1126.

Von Korff M, Wang H, Léon J and Pillen K (2004). Development of candidate introgression lines using an exotic barley accession ( Hordeum vulgare ssp. spontaneum) as donor. Theor. Appl. Genet. 109: 1736-1745.

Wang J, Wan X, Crossa J, Crouch J, et al. (2006). QTL mapping of grain length in rice (Oryza sativa L.) using chromosome segment substitution lines. Genet. Res. 88: 93-104.

Whipple CJ, Hall DH, DeBlasio S, Taguchi-Shiobara F, et al. (2010). A conserved mechanism of bract suppression in the grass family. Plant Cell 22: 565-578.

Xu YB (2010). Molecular Plant Breeding. CAB International, 195-248.

Yamanaka N, Watanabe S, Toda K, Hayashi M, et al. (2005). Fine mapping of the FT1 locus for soybean flowering time using a residual heterozygous line derived from a recombinant inbred line. Theor. Appl. Genet. 110: 634-639.

Yan JB, Tang H, Huang YQ, Zheng YL, et al. (2006). Quantitative trait loci mapping and epistatic analysis for grain yield and yield components using molecular markers with an elite maize hybrid. Euphytica 149: 121-131.

Young ND and Tanksley SD (1989). Restriction fragment length polymorphism maps and the conceptof graphical genotypes. Theor. Appl. Genet. 77: 95-101.

Zeng RZ, Akshay T, Liu F and Zhang GQ (2006). Mapping of the QTL for grain shape using single segment substitution lines in rice. Sci. Agric. Sin. 39: 647-654.

Zhao P, Liu RX, Li CP. Xing XR, et al. (2011). QTL mapping for grain yield associated traits using Ye478 introgression lines in maize. Sci. Agric. Sin. 44: 3508-3519.

Zhao FM, Zhang GQ, Zeng RZ, Yang ZL, et al. (2012). Epistatic and additive effects of QTL for yield-related traits using single segment substitution lines of rice (Oryza sativa L.). Acta Agron. Sin. 38: 2007-2014. 